\title{
Plasminogen Activator Inhibitor-1 Is
}

\section{Associated with Impaired Endothelial Function in Women with Systemic Lupus Erythematosus}

\author{
EMILY C. SOMERS $,{ }^{a}, b$ WENDY MARDER,${ }^{a}$ MARIANA J. KAPLAN,${ }^{a}$ \\ ROBERT D. BROOK, ${ }^{c}$ AND W. JOSEPH MCCUNE ${ }^{a}$ \\ ${ }^{a}$ University of Michigan, ${ }^{a}$ Division of Rheumatology, ${ }^{c}$ Division of Cardiology, \\ Ann Arbor, Michigan 48109, USA \\ ${ }^{b}$ London School of Hygiene \& Tropical Medicine, Infectious Disease Epidemiology Unit, \\ London WCIE 7HT, UK
}

\begin{abstract}
Endothelial function, measured noninvasively by brachial artery flow-mediated dilatation (FMD), has been shown to be impaired in patients with systemic lupus erythematosus (SLE). We hypothesized that depressed FMD in SLE patients is associated with increased levels of plasminogen activator inhibitor-1 (PAI-1), an inhibitor of fibrinolysis and regulator of vasoactivity. In this cross-sectional study of female SLE patients under the age of 55, putative markers of cardiovascular disease (CVD) such as PAI-1 were measured in addition to lupus-related disease activity (SLEDAI). The primary outcome, FMD, was measured using high-resolution ultrasound of the brachial artery gated to the $R$ wave to determine endothelial-dependent vasomotion. Endothelial-independent vasomotion was measured in response to nitroglycerin (NMD). Seventy-six female SLE patients, mean age $38.3 \pm 9.4$ years, were included. All patients demonstrated normal NMD responses, indicating that depression of FMD was related to decreased endothelial nitric oxide production. Increased PAI-1 was related to depressed FMD by univariate regression $(P=0.004)$. In a multivariable regression model adjusting for t-PA (tissue plasminogen activator)/PAI-1 ratio, SLEDAI, age at visit, family history of cardiovascular disease, SLE disease duration and body mass index, every $1 \mathrm{ng} / \mathrm{mL}$ increase in PAI-1 was associated with a reduction of 0.07 units FMD $(P=$ 0.039). PAI-1 was associated with impaired endothelial dysfunction, after controlling for several potential confounders. Given the high incidence of cardiovascular disease in SLE, further investigation of the role of subclinical markers of CVD is needed.
\end{abstract}

KeYwORDs: systemic lupus erythematosus (SLE); plasminogen activator inhibitor-1 (PAI-1); endothelium; flow-mediated dilatation

Address for correspondence: Emily C. Somers, University of Michigan Health System, Division of Rheumatology, 1150 W. Medical Center Dr., 5520 MSRB 1, Ann Arbor, MI 48109-0680. Voice: 734-936-1166; fax: 734-763-4151.

emsomers@umich.edu

Ann. N.Y. Acad. Sci. 1051: 271-280 (2005). (C) 2005 New York Academy of Sciences.

doi: 10.1196/annals.1361.068 


\section{BACKGROUND}

Morbidity and mortality due to cardiovascular disease (CVD) was recognized in systemic lupus erythematosus (SLE) patients as early as 1939, when Soma Weiss wrote "In this disease myocardial failure of varying degrees is rather common... the skin manifestations may be slight, preterminal, or even completely absent and the heart failure dominates the picture.... Histologic examination may reveal either no changes or interstitial edema, scarring, and at times thrombosis of the coronary arterioles." 1 More recently, a bimodal mortality pattern has been recognized in the SLE population, whereby a substantial number of late SLE deaths are related to CVD, in contrast to early deaths which are ascribed to active lupus and concomitant infections. ${ }^{2}$ Estimates of the increased risk for development of CVD in lupus patients range from an overall 8- to 9-fold increase, to a 50-fold increase in young women. ${ }^{3}$ Vascular insults characteristic of SLE are likely to underlie part of the excess risk of CVD in this population.

The plasminogen activation (PA) system constitutes a critical response mechanism to vascular injury. The central reaction of this system is the conversion of plasminogen to plasmin by tissue-type (t-PA) and urinary-type (u-PA) plasminogen activators. Plasminogen activator inhibitor 1 (PAI-1), a member of the serpin superfamily of protease inhibitors, plays a key role in regulating the PA system by serving as the primary inhibitor of t-PA and u-PA. PAI-1 also directly inhibits plasmin and appears to play a major role in determining the proliferative response to vascular injury by inhibiting the degradation of fibrin and several extracellular matrix proteins by plasmin. PAI-1 may also modulate vascular disease progression by plasminindependent mechanisms. ${ }^{4}$

As reviewed by Lyon and Hsueh, clinical studies have reported that circulating PAI1 levels are correlated with cardiovascular events, including those occurring among specific populations such as diabetes patients. ${ }^{5}$ An epidemiologic study based in Sweden demonstrated that increased PAI-1 concentrations increased risk of future incident myocardial infarctions. ${ }^{6}$ These relationships need to be confirmed in the lupus population. However, defects in the fibrinolytic system, such as decreased tissue-type plasminogen activator (t-PA) activity and elevated levels of PAI-1 in patients with SLE have been associated with an increased tendency to thrombosis. ${ }^{7,8}$ In addition, autoantibodies to recombinant PAI-1 have been described in SLE and correlate with clinical parameters measured by the BILAG disease activity index including global clinical score. Further, autoantibodies to fibrin-bound t-PA have been proposed to play a role in the pathogenesis of vascular disease in some SLE patients. ${ }^{9-11}$

This study was undertaken to characterize endothelial function in SLE patients, and to examine whether PAI-1 is associated with impaired flow-mediated dilatation, a measure of endothelial dysfunction, in young to middle-aged women with SLE.

\section{PATIENTS AND METHODS}

\section{Study Population}

A cross-sectional study of women with SLE was performed to examine markers of cardiovascular disease. Patients were recruited from the Michigan Lupus Cohort 
and University of Michigan outpatient rheumatology clinics. SLE patients fulfilling at least four American College of Rheumatology criteria for SLE, ${ }^{12,13}$ and aged less than 55 years, were invited to participate in the cardiovascular assessment. Patients were excluded if they were current smokers (within the previous 6 months), had a personal history of cardiovascular disease, thrombosis, or diabetes, were pregnant, or had a current infection. Acute infections may perturb endothelial function, and have been shown to be associated with a transient increase in risk of vascular events. ${ }^{14}$ Patients taking more than one antihypertensive medication in addition to a diuretic were also excluded as a safety precaution, since study participants were asked to postpone all medications on the morning of the study until after the cardiovascular assessments were performed. This research was approved by the University of Michigan Institutional Review Board, and all study participants signed written, informed consent forms.

\section{Study Procedures}

Study visits were performed at the University of Michigan General Clinical Research Center, in a temperature- and light-controlled room. To control for circadian variations, all visits were performed during morning hours. Patients were asked to fast, refrain from physical activity, and postpone any medications the morning of the visit until all functional assessments had been performed. Patients underwent vascular function measurements prior to venipuncture for the procurement of blood samples.

Data collection: Patients had a medical history and physical examination conducted by a rheumatologist, which included assessment of SLE activity as measured by the Systemic Lupus Erythematosus Disease Activity Index (SLEDAI). ${ }^{15}$ The Systemic Lupus International Collaborating Clinics/American College of Rheumatology (SLICC/ACR) Damage Index was used to evaluate accumulated damage (of at least 6 months' duration) owing to previous lupus activity, treatment, or intercurrent illness. ${ }^{16}$ Height and weight were measured with stadiometers and calibrated scales. Body mass index (BMI) was calculated as weight $(\mathrm{kg}) /$ height squared $\left(\mathrm{m}^{2}\right)$. BMI was categorized in accordance with guidelines from the obesity task force of the National Heart, Lung and Blood Institute (NHLBI), as follows: underweight $<18.5 \mathrm{~kg} / \mathrm{m}^{2}$, normal $18.5-24.9 \mathrm{~kg} / \mathrm{m}^{2}$, overweight $25-29.9 \mathrm{~kg} / \mathrm{m}^{2}$, obese I $30-$ $34.9 \mathrm{~kg} / \mathrm{m}^{2}$, obese II $35-39.9 \mathrm{~kg} / \mathrm{m}^{2}$, obese III $\geq 40 \mathrm{~kg} / \mathrm{m}^{2} .{ }^{17}$ Family history of cardiovascular disease was considered to be positive if a first-degree relative had experienced a cardiovascular event at age less than 55 or 65 years for male and female relatives, respectively.

Measurement of vascular function: Flow-mediated dilation (FMD) and nitroglycerin-mediated dilation (NMD) were measured noninvasively by ultrasound, with the study participant resting in the supine position. A $10-\mathrm{mHz}$ linear array transducer and an HP Image Point ultrasound system (Hewlett Packard, Andover, MA) were used for image acquisition. Baseline measurements of the brachial artery diameter were taken. Subsequently, a blood pressure cuff was inflated to $50 \mathrm{mmHg}$ above the participant's systolic blood pressure over the proximal portion of the right forearm for 4 minutes. FMD was determined 1 minute after release of the cuff. Brachial artery diameter was allowed to then return to baseline over a period of 15 minutes. To evaluate endothelium-independent nitric oxide responsiveness, $0.4 \mathrm{mg}$ nitroglyc- 
erin was administered sublingually; brachial artery images were obtained 3 minutes later. Serial longitudinal images of the brachial artery were obtained with image acquisition gated to the $\mathrm{R}$ wave from the electrocardiogram (24 sequential frames). Measurements were performed by an individual who was unaware of the clinical status of the participants. Individual frames were assessed utilizing a standard program (Brachial Analyzer with DICOM, Medical Imaging Applications LLC, Iowa City, IA). Only frames in which the image clarity was sufficient to ensure an accurate measurement were included. The end point of measurement was the percentage of change in mean brachial diameter in response to reactive hyperemia (FMD) or to nitroglycerin (NMD). The reproducibility of this technique in our unit has been reported previously. ${ }^{18,19}$

Assays: PAI-1 plasma levels were measured by an ELISA sandwich procedure using a solid-phase monoclonal antibody and an enzyme-labeled goat second antiserum for detection (IMUBIND plasma PAI-1 ELISA, American Diagnostica, Greenwich, CT). Tissue-plasminogen activator (t-PA) was measured in plasma using a double antibody ELISA (IMUBIND t-PA ELISA, American Diagnostica, Greenwich, CT). The assay utilizes human single chain t-PA as a standard calibrated against an international standard (NIBSAC, Hertfordshire, England). Assays for fibrinolytic markers were performed at the Medical Research Laboratories (Highland Heights, KY). In previous quality-control assessments performed by members of our research team, the monthly interassay coefficients of variation were found to be 4-9\% for PAI-1 and 3.8-8.7\% for t-PA. Medical Research Laboratories has been certified by the National Heart Lung and Blood Institute, Centers for Disease Control Part III program. ${ }^{20}$

Statistical analysis: Baseline summary statistics were computed as mean \pm standard deviation for continuous variables, or frequency and proportion for categorical variables. Variables were assessed for normality and log-transformed if appropriate. Pearson correlation coefficients were calculated to examine the strength and direction of associations between continuous variables. To estimate the effect of PAI- 1 on vascular function, univariate linear regression was performed. Multivariable regression was performed to account for potential confounders. $P$ values less than 0.05 were considered statistically significant. Data management and analysis were performed using Stata version 8 (StataCorp, College Station, TX).

\section{RESULTS}

Seventy-six female SLE patients participated in this research. In accordance with eligibility criteria, none of the study participants had previously experienced a cardiovascular event. Baseline characteristics of the population are presented in TABLE 1. Overall, lupus disease activity was relatively low among patients at the time of the study visit (mean SLEDAI score 3.6 \pm 3.8 ). Although average disease duration was long ( $12 \pm 7.2$ years), accumulated damage was notably low (ACR/SLICC damage score $1.1 \pm 1.8$ ). The majority of patients $(71 \%)$ had no family history of premature cardiovascular disease in a first-degree relative.

The primary outcome measure, FMD, was normally distributed with a mean of $4.0 \pm 4.6 \mathrm{SD}$ (range, 8.8 to $13.5 \%$ ). All patients demonstrated normal NMD responses, suggesting that cases of FMD impairment were related to deficits in endothelial 
TABLE 1. Baseline characteristics of the study population

\begin{tabular}{lc}
\hline & SLE Patients $(n=76)$ \\
\hline Female & $100 \%$ \\
Age at visit & $38.3 \pm 9.4$ \\
Race/ethnicity & $65(86 \%)$ \\
White & $10(13 \%)$ \\
Black & $1(1 \%)$ \\
Hispanic & \\
Body Mass Index $\left(\mathrm{kg} / \mathrm{m}^{2}\right)$ & $2(2.6 \%)$ \\
Underweight $(<18.5)$ & $39(51.3 \%)$ \\
Normal $(18.5-24.9)$ & $21(27.6 \%)$ \\
Overweight $(25-29.9)$ & $8(10.5 \%)$ \\
Obese I $(30-34.9)$ & $2(2.6 \%)$ \\
Obese I $(35-39.9)$ & $4(5.3 \%)$ \\
Obese III $(\geq 40)$ & \\
Family history of CVD & $54(71 \%)$ \\
No & $19(25 \%)$ \\
Yes & $3(4 \%)$ \\
Unknown & $12 \pm 7.2$ \\
Duration of SLE $(\mathrm{yr})$ & $1.1 \pm 1.8$ \\
SLICC damage score & $3.6 \pm 3.8$ \\
SLEDAI score & $4.0 \pm 4.6$ \\
FMD $(\%)$ & $20.2 \pm 19$ \\
PAI-1 (ng/mL) & $7.9 \pm 9.1$ \\
t-PA (ng/mL) & \\
\hline
\end{tabular}

Note: Data expressed as mean \pm SD or frequency $(\%)$.

nitric oxide production rather than altered responses of arterial smooth muscle to nitric oxide. PAI-1 levels were negatively correlated with FMD $(r=-0.33, P=0.004)$. When PAI-1 was categorized according to quartile, mean FMD ranged from $5.3 \%$ for the lowest PAI-1 quartile to $2.7 \%$ for the highest PAI-1 quartile (FIG. 1). Increased PAI-1 was related to depressed FMD by univariate regression; for each $1 \mathrm{ng} / \mathrm{mL}$ increase in PAI-1 there was a corresponding reduction of 0.08 units FMD $(P=0.004)$. A similar association was demonstrated by multivariable regression modeling. When adjusted for age at visit, disease duration, SLEDAI score, t-PA/PAI-1 ratio, family history of cardiovascular disease, and body mass index, each increase in $1 \mathrm{ng} /$ $\mathrm{mL}$ of PAI- 1 was associated with a reduction of 0.07 units FMD $(P=0.039)$. PAI-1 was the only variable in the model that was significantly associated with FMD, and there was no evidence for interaction between variables. 


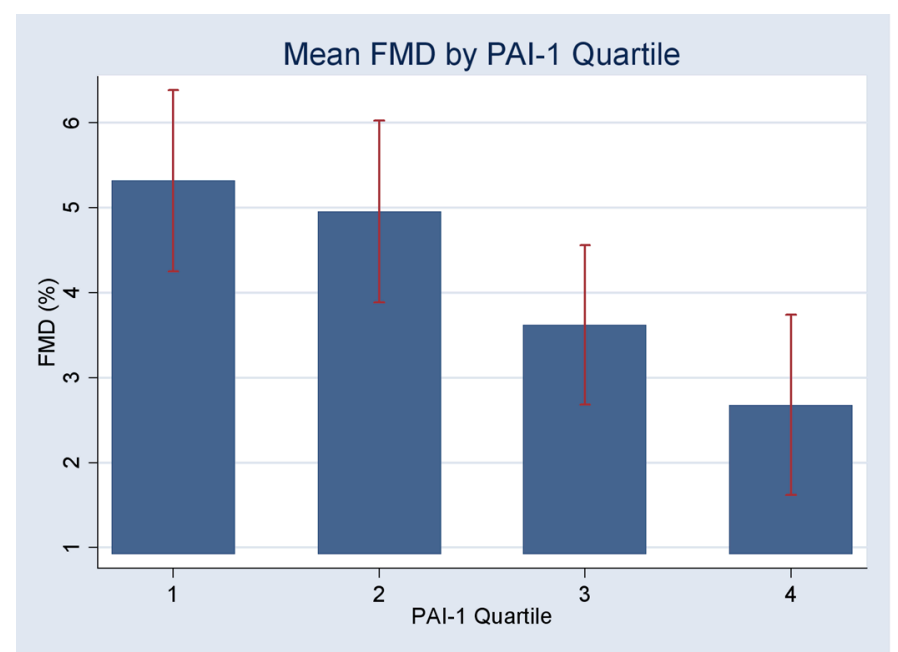

FIGURE 1. Increases in PAI-1 levels are associated with decreased flow-mediated dilatation. Bars represent mean FMD \pm SEM, according to quartile of PAI-1 $\left(\mathrm{P}_{\text {trend }}=0.044\right)$.

\section{DISCUSSION}

In this ongoing study, we found an inverse relationship between circulating PAI1 levels and endothelial function, as measured by flow-mediated dilatation, in women under 55 years old with SLE. This relationship persisted after controlling for several potential confounders, including age, BMI, and lupus disease activity.

Relatively small reductions in brachial FMD, similar to those which we reported between the lowest and highest PAI-1 quartiles in this study, have been shown to be independently associated with worse cardiovascular prognosis. ${ }^{21}$ Although formal cutoffs have not been established for normal versus abnormal FMD, and calibrations in various laboratories can be different, our research group considers less than $4 \%$ to represent impaired endothelial function. A full $47 \%$ of the SLE population in this study had an FMD value of less than 4\%, indicating that a substantial proportion of these patients exhibit some degree of endothelial dysfunction. Within this group of patients exhibiting low FMD, further cardiovascular risk profiling may help identify pathogenic mechanisms and potential strategies to forestall development of clinically significant vascular damage.

A link between PAI-1 and endothelial dysfunction is biologically plausible. PAI1 appears to play a crucial role in fibrin homeostasis, with diminished plasmin generation being the primary downstream mediator of PAI-1's effect. ${ }^{22}$ PAI-1 has been hypothesized to play an important role in regulating thrombin activity in the blood vessel wall, an environment in which thrombin may stimulate VSMC proliferation. ${ }^{23-26}$ Furthermore, PAI-1 could potentially promote interactions between vascular cells and the extracellular matrix. ${ }^{22}$ In addition, it has been proposed that 
upregulation of PAI-1 expression after arterial injury favors atherosclerosis development. ${ }^{22}$ Conversely, in mice that completely lack PAI-1, the lysis of arterial thrombi is accelerated and thrombus formation after arterial injury is delayed. ${ }^{24,27,28}$

A cause-and-effect relationship has not been established between vascular PAI-1 expression and the size and extent of atherosclerotic lesions. Elevated plasma PAI-1 levels are associated with complications of atherosclerosis. By affecting VSMC migration, PAI-1 could modulate the composition of plaques and their susceptibility to acute complications. Therefore, it is probable that PAI-1 promotes atherogenesis and/or its complications in humans, although there are insufficient data at this time to make definitive conclusions. ${ }^{22}$

PAI-1 and its regulation may have particular relevance in lupus. The PAI-1 4G/ $4 \mathrm{G}$ genotype has been proposed to influence the development of lupus nephritis. The $4 \mathrm{G} / 5 \mathrm{G}$ polymorphism of the PAI-1 gene was reported to be associated with the activity but not the chronicity of lupus nephritis. The presence of the $4 \mathrm{G} 4 \mathrm{G}$ genotype did not increase the risk of developing SLE or lupus nephritis, but predicted the development of higher nephritis activity and more extensive necrotizing lesions. ${ }^{29}$ Further, the presence of the $4 \mathrm{G}$ allele of the $4 \mathrm{G} / 5 \mathrm{G}$ polymorphism of the PAI- 1 gene has been proposed as an additional risk factor for the development of arterial thrombosis in antiphospholipid antibody syndrome. ${ }^{29,30}$ In addition, osteonecrosis in SLE has been reported to be significantly associated with elevated levels of PAI-1 activity and with elevated PAI-1/t-PA ratio. ${ }^{31}$

Glomerular PAI-1/t-PA balance is involved in extracellular matrix turnover, as well as fibrin deposition in glomeruli. PAI-1 mRNA levels are significantly increased in MRL/lpr and NZB/NZW animals compared with controls. PAI-1 mRNA or protein is not found in normal mouse or human kidney, but is strongly upregulated in MRL/lpr mouse kidneys, as well as many other experimental and human renal diseases. This and other studies have suggested that changes in the expression of these genes may promote the formation of microthrombi and thus contribute to the progression of lupus nephritis. Increased TGF-1 expression has been reported in murine and human lupus nephritis and this molecule stimulates PAI-1 synthesis. PAI-1 has been implicated in extracellular matrix accumulation in kidney by its effects to inhibit matrix degradation. ${ }^{32-39}$

The association between PAI-1 and depressed FMD that we have found in this study further implies a role for PAI-1 in endothelial function among lupus patients. Coupled with previous findings from our group demonstrating an association between apoptotic endothelial cells and both endothelial dysfunction and plasma tissue factor, multiple candidate risk factors for cardiovascular disease in SLE are emerging. ${ }^{40}$ Whether abnormalities or interactions of these biomarkers are unique to SLE, and the degree to which they contribute to vascular injury and CVD risk, remain to be elucidated. It will be important within a larger series of lupus patients and controls to analyze all of these factors in concert, in order to more fully characterize the role of these markers and potential interactive effects.

The current study has several limitations. First, due to the cross-sectional design, we were not able to assess the temporality of the association between PAI-1 and FMD. Further, while the utility of FMD has been shown in other populations, we will need long-term follow-up to determine the predictive value of FMD in these lupus patients in terms of future cardiovascular events. It will also be informative to study the association of fibrinolysis and endothelial function in non-lupus controls. Due to 
the relatively small size of the population in this study, we were limited in terms of statistical power to perform more detailed subset analyses at this time.

We performed all study measurements during the morning hours to account for circadian variation in PAI-1 levels; plasma concentrations have been observed to be lower during the day versus night. ${ }^{41}$ However, despite the fact that we performed vascular measurements in a controlled environment to the extent possible, for logistical reasons we were unable to time study assessments according to the menstrual cycle. This would have been preferable, as variation of PAI-1 and t-PA has been reported within the menstrual cycle, with higher values outside of days 2-7 of the menstrual cycle. ${ }^{42}$

Features underlying lupus pathology are hypothesized to play a key role in vascular injury. We have identified PAI-1 as a potential marker of increased risk, and future investigations should be performed to discern whether PAI-1 is directly involved in the etiology of CVD in lupus. We are continuing study enrollment, which will enable further subset analyses, and the characterization of additional biomarkers in a larger population. This may provide further elucidation of mechanisms of endothelial injury in systemic lupus, which would aid in the development of targeted interventions.

\section{ACKNOWLEDGMENTS}

This work was funded by the Arthritis Foundation, Lupus Research Institute, Herbert and Carol Amster Lupus Fund, Klein Lupus Research Fund, and the University of Michigan General Clinical Research Center (GCRC) (Grant M01-RR00042)

\section{REFERENCES}

1. Weiss, S. 1939. Diseases of the heart and the aorta which are not well recognized. Med. Clin. North Am. 5: 1323-1344.

2. URowitz, M.B. \& D.D. Gladman. 1980. Late mortality in SLE: "the price we pay for control." J. Rheumatol. 7: 412-416.

3. Manzi, S., F. Selzer, K. Sutton-Tyrrell, et al. 1999. Prevalence and risk factors of carotid plaque in women with systemic lupus erythematosus. Arthritis Rheum. 42: $51-60$.

4. FAy, W.P., D.T. EITZMan, A.D. Shapiro, et al. 1994. Platelets inhibit fibrinolysis in vitro by both plasminogen activator inhibitor-1-dependent and -independent mechanisms. Blood 83: 351-356.

5. LyON, C.J. \& W.A. HsueH. 2003. Effect of plasminogen activator inhibitor-1 in diabetes mellitus and cardiovascular disease. Am. J. Med. (Suppl.) 115: 62S-68S.

6. Thogersen, A.M., J.H. Jansson, K. Boman, et al. 1998. High plasminogen activator inhibitor and tissue plasminogen activator levels in plasma precede a first acute myocardial infarction in both men and women: evidence for the fibrinolytic system as an independent primary risk factor. Circulation 98: 2241-2247.

7. Gong, R., Z. LiU, Z. CHEN, et al. 2002. [Genetic variations in plasminogen activator inhibitor-1 gene and beta fibrinogen gene associated with glomerular microthrombosis in lupus nephritis and the gene dosage effect]. Zhonghua Yi Xue Yi Chuan Xue Za Zhi 19: 1-5.

8. BICK, R.L. 2000. Recurrent miscarriage syndrome due to blood coagulation protein/ platelet defects: prevalence, treatment and outcome results. DRW Metroplex Recurrent Miscarriage Syndrome Cooperative Group. Clin. Appl. Thromb. Hemost. 6: $115-125$. 
9. Bates, R.L., S.J. Payne, S.L. Drury, et al. 2003. The prevalence and clinical significance of autoantibodies to plasminogen activator inhibitor 1 in systemic lupus erythematosus. Lupus 12: 617-622.

10. Salazar-Paramo, M., I. Garcia de la Torre, M.J. Fritzler, et al. 1996. Antibodies to fibrin-bound tissue-type plasminogen activator in systemic lupus erythematosus are associated with Raynaud's phenomenon and thrombosis. Lupus 5: 275-278.

11. Ruiz-Arguelles, A., E. Angles-Cano, B. Perez-Romano, et al. 1995. Serum antibodies to distinct epitopes of the tissue-type plasminogen activator (t-PA) in patients with systemic lupus erythematosus. Am. J. Hematol. 49: 109-114.

12. TAN, E.M., A.S. CoHEN, J.F. FrIES, et al. 1982. The 1982 revised criteria for the classification of systemic lupus erythematosus. Arthritis Rheum. 25: 1271-1277.

13. Hochberg, M.C. 1997. Updating the American College of Rheumatology revised criteria for the classification of systemic lupus erythematosus. Arthritis Rheum. 40: 1725.

14. Smeeth, L., S.L. Thomas, A.J. Hall, et al. 2004. Risk of myocardial infarction and stroke after acute infection or vaccination. N. Engl. J. Med. 351: 2611-2618.

15. Bombardier, C., D.D. Gladman, M.B. Urowitz, et al. 1992. Derivation of the SLEDAI. A disease activity index for lupus patients. The Committee on Prognosis Studies in SLE. Arthritis Rheum. 35: 630-640.

16. Gladman, D., E. Ginzler, C. Goldsmith, et al. 1996. The development and initial validation of the Systemic Lupus International Collaborating Clinics/American College of Rheumatology damage index for systemic lupus erythematosus. Arthritis Rheum. 39: $363-369$.

17. Expert Panel on the Identification, Evaluation, and Treatment of Overweight IN ADULTS. 1998. Clinical guidelines on the identification, evaluation, and treatment of overweight and obesity in adults: executive summary. Am. J. Clin. Nutr. 68: 899917.

18. Rajagopalan, S., R. Brook, R.H. Mehta, et al. 2002. Effect of losartan in agingrelated endothelial impairment. Am. J. Cardiol. 89: 562-566.

19. BRook, R.D., J.R. BROOK, B. URCH, et al. 2002. Inhalation of fine particulate air pollution and ozone causes acute arterial vasoconstriction in healthy adults. Circulation 105: $1534-1536$.

20. Myers, G.L., G.R. Cooper, C.L. Winn, et al. 1989. The Centers for Disease ControlNational Heart, Lung and Blood Institute Lipid Standardization Program: an approach to accurate and precise lipid measurements. Clin. Lab. Med. 9: 105-135.

21. Lerman, A. \& A.M. Zeiner. 2005. Endothelial function: cardiac events. Circulation 111: $363-368$.

22. FAY, W.P. 2004. Plasminogen activator inhibitor 1, fibrin, and the vascular response to injury. Trends Cardiovasc. Med. 14: 196-202.

23. Peng, L., N. Bhatia, A.C. Parker, et al. 2002. Endogenous vitronectin and plasminogen activator inhibitor-1 promote neointima formation in murine carotid arteries. Arterioscler. Thromb. Vasc. Biol. 22: 934-939.

24. Farrehi, P.M., C.K. Ozaki, P. Carmeliet, et al. 1998. Regulation of arterial thrombolysis by plasminogen activator inhibitor-1 in mice. Circulation 97: 1002-1008.

25. FAY, W.P., J.G. MURPhY W.G. OWEN. 1996. High concentrations of active plasminogen activator inhibitor-1 in porcine coronary artery thrombi. Arterioscler. Thromb. Vasc. Biol. 16: 1277-1284.

26. Zhu, Y., P.M. Farrehi \& W.P. Fay. 2001. Plasminogen activator inhibitor type 1 enhances neointima formation after oxidative vascular injury in atherosclerosisprone mice. Circulation 103: 3105-10.

27. Zhu, Y., P. CARMEliet \& W.P. FAY. 1999. Plasminogen activator inhibitor-1 is a major determinant of arterial thrombolysis resistance. Circulation 99: 3050-3055.

28. FAy, W.P., A.C. PARKer, L.R. Condrey, et al. 1997. Human plasminogen activator inhibitor-1 (PAI-1) deficiency: characterization of a large kindred with a null mutation in the PAI-1 gene. Blood 90: 204-208.

29. WANG, A.Y., P. PoON, F.M. LAI, et al. 2001. Plasminogen activator inhibitor-1 gene polymorphism 4G/4G genotype and lupus nephritis in Chinese patients. Kidney Int. 59: $1520-1528$. 
30. Tassies, D., G. Espinosa, F.J. Munoz-Rodriguez, et al. 2000. The 4G/5G polymorphism of the type 1 plasminogen activator inhibitor gene and thrombosis in patients with antiphospholipid syndrome. Arthritis Rheum. 43: 2349-2358.

31. Sheikh, J.S., G.S. Retzinger \& E.V. Hess. 1998. Association of osteonecrosis in systemic lupus erythematosus with abnormalities of fibrinolysis. Lupus 7: 42-8.

32. BAO, L., J. Zhou, V.M. Holers, et al. 2003. Excessive matrix accumulation in the kidneys of MRL/lpr lupus mice is dependent on complement activation. J. Am. Soc. Nephrol. 14: 2516-2525.

33. Hamano, K., M. Iwano, Y. Akai, et al. 2002. Expression of glomerular plasminogen activator inhibitor type 1 in glomerulonephritis. Am. J. Kidney Dis. 39: 695-705.

34. Yamamoto, T., N.A. Noble, A.H. Cohen, et al. 1996. Expression of transforming growth factor-beta isoforms in human glomerular diseases. Kidney Int. 49: 461-469.

35. Yамамото, K. \& D.J. LoskutofF. 1997. The kidneys of mice with autoimmune disease acquire a hypofibrinolytic/procoagulant state that correlates with the development of glomerulonephritis and tissue microthrombosis. Am. J. Pathol. 151: 725734.

36. Yамамото, K. \& D.J. Loskutoff. 2000. Expression of transforming growth factorbeta and tumor necrosis factor-alpha in the plasma and tissues of mice with lupus nephritis. Lab. Invest. 80: 1561-1570.

37. Keeton, M., C. Ahn, Y. Eguchi, et al. 1995. Expression of type 1plasminogen activator inhibitor in renal tissue in murine lupus nephritis. Kidney Int. 47: 148-157.

38. Troyer, D.A., B. Chandrasekar, T. Thinnes, et al. 1995. Effects of energy intake on type 1 plasminogen activator inhibitor levels in glomeruli of lupus-prone $\mathrm{B} / \mathrm{W}$ mice. Am. J. Pathol. 146: 111-120.

39. Moll, S., P.A. Menoud, T. Fulpius, et al. 1995. Induction of plasminogen activator inhibitor type 1 in murine lupus-like glomerulonephritis. Kidney Int. 48: 1459-1468.

40. Rajagopalan, S., E.C. Somers, R.D. BROOK, et al. 2004. Endothelial cell apoptosis in systemic lupus erythematosus: a common pathway for abnormal vascular function and thrombosis propensity. Blood 103: $3677-3683$.

41. Angleton, P., W.L. Chandler \& G. Schmer. 1989. Diurnal variation of tissue-type plasminogen activator and its rapid inhibitor (PAI-1). Circulation 79: 101-106.

42. Sowers, M., C. Derby, M.L. Jannausch, et al. 2003. Insulin resistance, hemostatic factors, and hormone interactions in pre- and perimenopausal women: SWAN. J. Clin. Endocrinol. Metab. 88: 4904-4910. 